\title{
Time Symmetry and the Many-Worlds Interpretation
}

\author{
Lev Vaidman
}

\begin{abstract}
An attempt to solve the collapse problem in the framework of a time-symmetric quantum formalism is reviewed. Although the proposal does not look very attractive, its concept - a world defined by two quantum states, one evolving forwards and one evolving backwards in time - is found to be useful in modifying the many-worlds picture of Everett's theory.
\end{abstract}

\section{Introduction}

Quantum mechanics is an almost unprecedented success as a physical theory, yielding precise predictions for the results of experiments. However, if quantum theory is viewed as a direct description of physical reality, there is a significant difficulty: in order to explain particular outcomes of quantum measurements, a collapse of the quantum state has to be introduced. The collapse, with its randomness, nonlocality and the lack of a well defined moment of occurrence, is such an ugly scar on quantum theory, that I, along with many others, am ready to follow Everett and deny its existence. The price is the Many Worlds Interpretation (MWI), i.e., the existence of numerous parallel worlds.

There are other attempts to avoid collapse. One of them is a proposal due to Aharonov [1], according to which, in addition to the standard forwards evolving quantum state, there is a backwards evolving quantum state. Both evolve according to the Schrödinger equation without collapses, but a particular form of the backwards evolving state ensures that we do not experience multiple branches of the forwards evolving state, just one. The form of the backwards evolving state is chosen (somewhat artificially) exactly by 
this requirement: that each quantum measurement ends up with a single outcome.

There is a certain difference between the single world described by quantum mechanics with collapses at each measurement, and the single world which emerges with the backwards evolving quantum state of Aharonov. While the former, at each moment in time, is defined by the results of measurements in the past, the latter is defined in addition by the results of a complete set of measurements in the future. Although I am not ready to accept Aharonov's proposal, I think that his idea of a single world emerging from forwards and backwards evolving quantum states is useful. I will argue that there is an advantage in drawing the Everettian many worlds picture using multiple Aharonov worlds, instead of multiple worlds generated solely by quantum measurements in the past.

I will start with a brief description of the time-symmetric Two-State Vector Formalism (TSVF) [2], which provides the framework for Aharonov's proposal.

\section{The Two-State Vector Formalism}

The TSVF describes quantum systems at a given time by a backwards evolving quantum state [3], in addition to the standard, forwards evolving quantum state. An ideal (text book) quantum measurement at time $t$ of a variable $A$ with an outcome $a$ creates a quantum state $|A=a\rangle$ evolving forwards in time and, at the same time, creates a quantum state evolving backwards in time, towards the past, which we denote $\langle A=a|$. An ideal measurement also serves as a verification measurement: the outcome $A=a$ is obtained with probability 1 if the state $|A=a\rangle$ evolves towards time $t$ from the past or (and) if the state $\langle A=a|$ evolves towards time $t$ from the future.

In a real laboratory we usually have separate devices for the creation and verification of quantum states: emitters and detectors. We can place a single photon source (made today in a number of laboratories around the world) in front of a two-slit barrier. It creates quantum states of photons exhibiting an interference pattern, which can be tested by measuring the frequency of clicks of a photodetector as a function of its position on the plain located beyond the barrier (see Figure 1a). The backwards evolving quantum state of the photon is created by the detector and it is tested by the source. We can observe the interference pattern of the backwards evolving state by fixing 

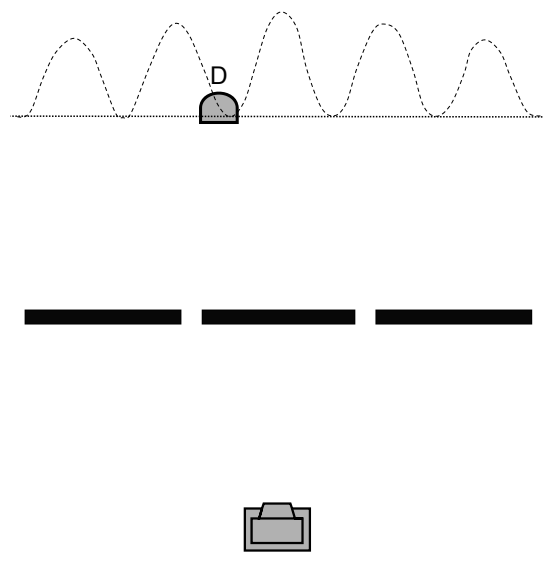

$\mathrm{S}$

(a) The number of absorbed particles as a function of the position of a detector shows the interference pattern of the forwards evolving quantum state.
D

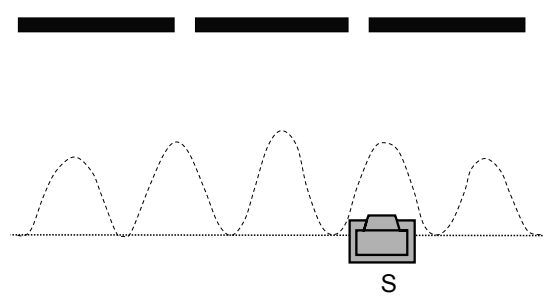

(b) The number of emitted particles absorbed by the detector, as a function of the position of the source, shows the interference pattern of the backwards evolving quantum state.

Figure 1: Interference patterns

the position of the photodetector in front of the slits and moving the source along the plane parallel to the barrier. The frequency of emitted photons, post-selected by the condition of being observed by the detector, exhibits the familiar two-slits interference pattern, but this time it is interference of the backwards evolving state (see Figure 1b).

Somewhat surprisingly, we do have numerous realizations of essentially ideal von Neumann measurements in Nature: the measuring device is the environment and the measured systems are macroscopic objects or, sometimes, charged particles. Interactions with molecules, photons, phonons, etc. of the environment provide nondemolition localization measurements.

Given complete measurements preparing the state $|\Psi\rangle$ before time $t$ and complete measurements verifying the state $|\Phi\rangle$ after time $t$, the quantum system at time $t$ is described by the two-state vector [4]:

$$
\langle\Phi|| \Psi\rangle
$$

The two-state vector provides maximal information regarding the way the quantum system can affect at time $t$ any other system. In particular, 
the two-state vector describes the influence on a measuring device coupled with the system at time $t$. An ideal measurement of a variable $O$ yields an eigenvalue $o_{n}$ with probability given by the Aharonov, Bergman, Lebowitz $(\mathrm{ABL})$ rule [5]:

$$
\operatorname{Prob}\left(o_{n}\right)=\frac{\left|\left\langle\Phi\left|\mathbf{P}_{O=o_{n}}\right| \Psi\right\rangle\right|^{2}}{\sum_{j}\left|\left\langle\Phi\left|\mathbf{P}_{O=o_{j}}\right| \Psi\right\rangle\right|^{2}} .
$$

An important case is when a particular measurement has an outcome that occurs with certainty. In this case I call it (somewhat misleadingly) an element of reality [7].

The most important result of the TSVF has been the discovery of weak values of physical variables [6]. When, at time $t$, another system couples weakly to a variable $O$ of a pre- and post-selected system $\langle\Phi|| \Psi\rangle$, the effective coupling is not to one of the eigenvalues, but to the weak value:

$$
O_{w} \equiv \frac{\langle\Phi|O| \Psi\rangle}{\langle\Phi \mid \Psi\rangle}
$$

Since the quantum states remain effectively unchanged during the measurement, several weak measurements can be performed one after another and even simultaneously. Weak-measurement elements of reality [8], i.e., the weak values, provide a self-consistent but sometimes very unusual picture for preand post-selected quantum systems.

As a simple example, consider a particle emitted from a source $S$ towards a beam splitter $B S$, and detected by a detector $A$ (see Figure 2). In our simplified model, the forwards evolving state corresponds to the line from the source towards the beam splitter, and then to the two lines from the beam splitter towards the two detectors. The backwards evolving state corresponds to the line from detector $A$ towards the beam splitter, and then to two lines, one towards the source and one towards nowhere. If we denote the quantum state evolving from the beam splitter towards detector $A$ as $|A\rangle$, and similarly, the state evolving from the beam splitter towards detector $B$ as $|B\rangle$, then the two-state vector of the particle, at a time when the particle has passed the beam splitter but has not reached the detectors, is:

$$
\left\langleA \left|\quad \frac{1}{\sqrt{2}}(|A\rangle+|B\rangle)\right.\right.
$$

From the ABL rule and from the calculation of the weak values of projections on various locations, we can immediately see that both strong and 


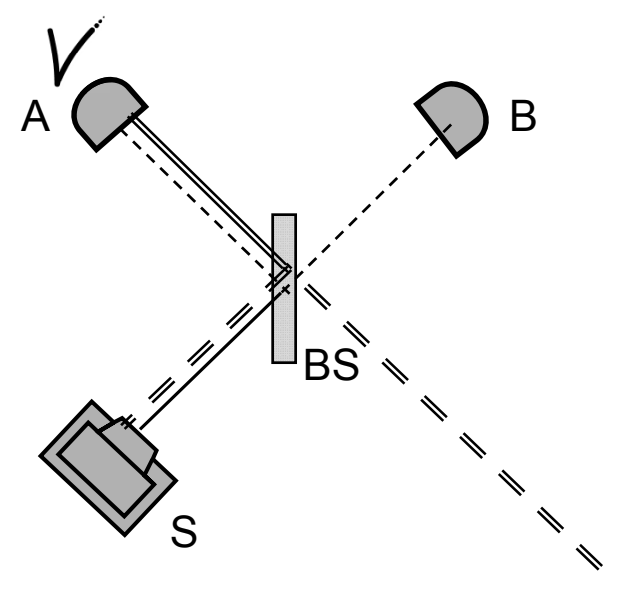

Figure 2: A particle is emitted by the source and absorbed by detector $A$. Single lines denote forwards and double lines denote backwards evolving quantum states.

weak-measurement elements of reality show that the particle is well localized during all of its motion along the trajectory $S-B S-A$. The weak-measurement elements of reality for projections on the corresponding trajectories are: $\left(\mathbf{P}_{A}\right)_{w}=1$ and $\left(\mathbf{P}_{B}\right)_{w}=0$.

A more entertaining example is the three-box paradox [9]. Consider a single particle in three boxes described by the two-state vector

$$
\frac{1}{3}(\langle A|+\langle B|-\langle C|) \quad(|A\rangle+|B\rangle+|C\rangle),
$$

where $|A\rangle$ is a quantum state of the particle located in box $A$, etc. For this particle, there is a set of elements of reality:

$$
\begin{aligned}
& \mathbf{P}_{A}=1, \\
& \mathbf{P}_{B}=1 .
\end{aligned}
$$

Or, in words: if we open box $A$, we find the particle there for sure; if we open box $B$ (instead), we also find the particle there for sure.

For this particle there are also corresponding weak-measurement elements of reality: $\left(\mathbf{P}_{A}\right)_{w}=1$ and $\left(\mathbf{P}_{B}\right)_{w}=1$. Any weak coupling to the particle 
in box $A$ behaves as if there is a particle there. And, simultaneously, the same is true for box $B$. Note that these are properties of neither forwards nor backwards evolving states separately, but only of both together.

\section{The two-state vector formalism without col- lapse}

It is uncontroversial to apply the concepts of the TSVF, such as the twostate vector, weak values, etc., to a quantum system in the past, when both pre- and post-selection have already taken place. The revolutionary proposal of Aharonov [1] is that the backwards evolving state exists at the present moment. Aharonov's backwards evolving state is a very special one. It ensures that all quantum measurements have definite outcomes. In particular, in our simple example with a beam splitter and a detector, the backwards evolving state has a component of triggered detector $A$ and does not have a component of triggered detector $B$. Thus, although the forwards evolving state includes a component in which the particle arrives at detector $B$, it will leave no trace. Any weak coupling with the particle on the trajectory $B S-B$ will show nothing.

I find Aharonov's proposal very problematic. It does remove action at a distance and randomness from basic physical interactions, two of the main difficulties with the collapse postulate. But it still has the third: it is not well defined. The backwards evolving quantum state needs to be tailored in such a way that all measurements will have a definite result, but what is the definition of a measurement?

The difficulty is increased by the fact that the backwards evolving state needs to be very specific. The backwards evolving state is specified by (vaguely defined) measurement events, whose probability of occurrence is given by the Born rule applied to the forwards evolving state. It will not be the case that the backwards evolving state alone describes well defined results of measurements. For consider a longer history which includes our experiment (Figure 3). Assume that the click of a detector $A$ causes a lamp to be switched on. Then, a photon from the lamp passes through another beam splitter and is detected by another detector $C$. A definite outcome for detector $C$ implies that only part of the photon's backwards evolving wave reaches detector $A$. Therefore, the backwards evolving quantum state is not 


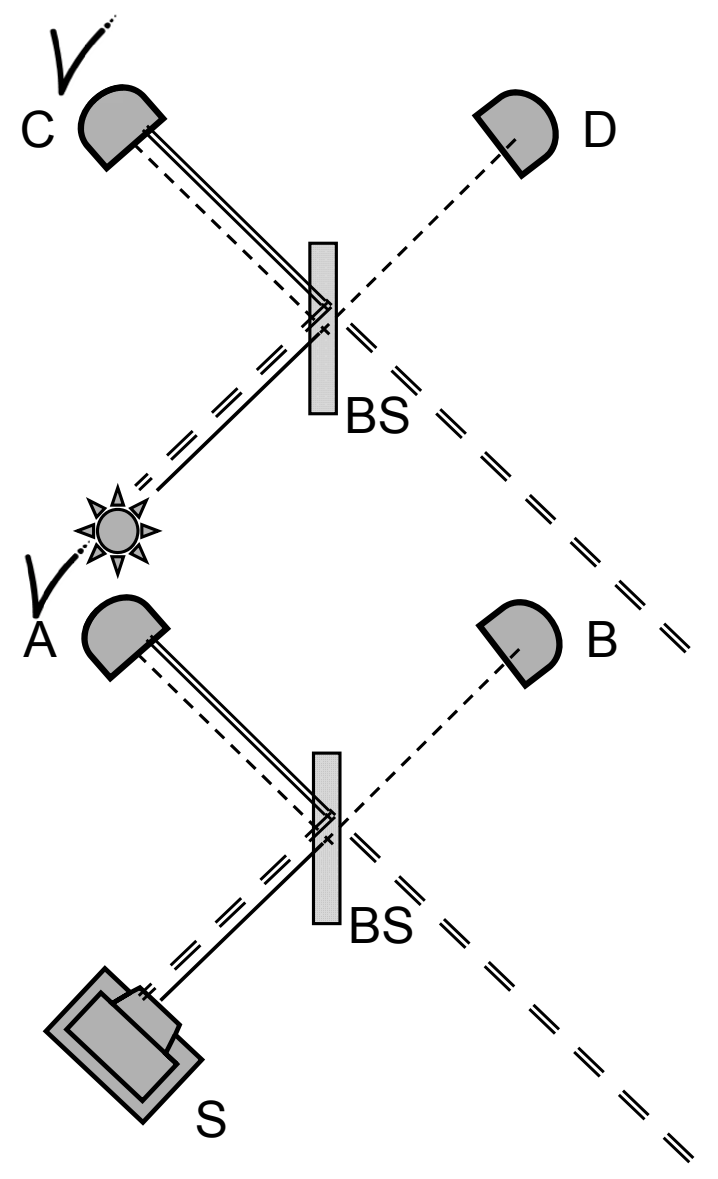

Figure 3: A particle emitted by the source is absorbed by detector $A$. It switches on the lamp and a photon from the lamp is absorbed by detector C. 
an eigenstate of the clicked detector $A$. Aharonov and Gruss [1] claim that it is feasible to arrange the backwards evolving state in such a way that, together with the forwards evolving state, only a single history of "macroscopic" events is significant. The weak value of the projection on the outcome $A$ is exponentially larger (in the number of particles in the detector) than the weak value of the projection on the outcome $B$. However, it is hard to see how Aharonov's program can succeed for a long lasting Universe, and very many measurement events in parallel worlds.

The artificiality of the definition of the backwards evolving quantum state together with difficulties in making this program consistent make me very reluctant to accept Aharonov's hypothesis that at each moment in time, there is a quantum state evolving backwards. But in the context of Everettian quantum theory, Aharonov's idea is useful. It is possible to describe each individual world (i.e., branch of the universe) in terms of both a forwards and a backwards evolving state, with the outcomes of both past and future measurements fixed. I find this preferable to the standard Everettian approach.

I will begin my argument with a brief description of my understanding of the concept of a world in the MWI.

\section{Everett "worlds"}

The basic concept in the MWI is a "world". It belongs not to the mathematical formalism of quantum mechanics, but to its interpretation. It helps to make a connection between the mathematical formalism and our experience.

One approach to the concept of a world (which seems to be very close to Everett's original proposal) is to define a subjective world for each observer - that is, a quantum state of the universe relative to a particular conscious state of the observer. This approach is certainly consistent. But it is not very effective, since it is hard to discuss objects which are not in a direct contact with the observer, e.g., objects in the far past, or the far future, or which are far away. We would like to discuss stars before life developed. Therefore, I prefer not to define concepts in terms of conscious observers. Instead, I define worlds in an objective way:

A world is the totality of (macroscopic) objects: stars, cities, people, grains of sand, etc. in a definite classically described 
state.

The MWI in Stanford Philosophy Encyclopedia [14]

I will now clarify (and slightly amend) this definition. First, a world is not a concept associated with a particular moment in time, but with all of time. It is a complete history in which all macroscopic objects have definite states. At the beginning, according to the standard MWI, there was one classical state, common to all worlds. At a later time there are multiple classically described states. Each one corresponds to numerous worlds with identical pasts and different futures.

The second clarification is that, apart from classical objects, the description of a world should include a description of some microscopic objects. A world is a sensible story, a causal connection between states of objects. We need not describe the quantum states of all the particles of a table, but the quantum state of a particle in an accelerator, which leaves a trace in a bubble chamber, or the state of a particle entering the beam splitter in one of the experiments described above, are certainly important parts of the "story" of a world. Strictly speaking, the outcomes of quantum measurements, recorded in the definite classically described states of parts of the measuring devices, define the quantum states of the particle and we can tell the story replacing the particle's evolution by the set of outcomes of macroscopic measuring devices. But this is very artificial, so we adopt a description of a world as a classical state of macroscopic objects and, in addition, quantum states of (a few) relevant microscopic objects.

A good example of a quantum system which requires description in a world is a single particle passing through a beam splitter. If the particle passes the first beam splitter of a properly tuned Mach-Zehnder interferometer (MZI, see Figure 4), its description as a superposition of being in two separate locations is necessary to explain why it will end up with certainty in one output of the interferometer and not in the other. If, after the first beam splitter, there are detectors, as in the experiment described in Figure 2, then we may say that we need to describe the particle as being in a superposition for another reason. We might bet on one of the outcomes, and in order to place an intelligent bet we have to know the quantum state of the particle.

There is a big difference between strongly interacting quantum particles, like charged particles in the detector of an accelerator, and weakly interacting particles, like photons in an interferometer. Strongly interacting particles, in the same way as macroscopic bodies, are frequently measured by the en- 


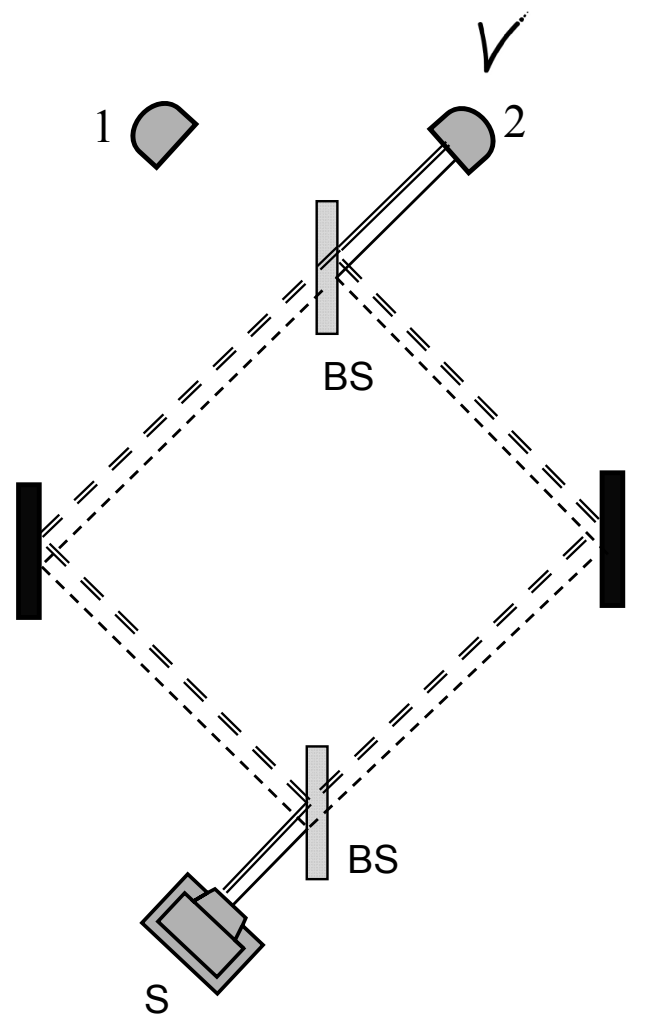

Figure 4: A Mach-Zehnder interferometer, tuned in such a way that all particles from $S$ go to the port 2 . 
vironment, or by special detectors like bubble chambers. Therefore, when a strongly interacting particle is included as part of a world, it is nearly always well localized, with a definite classical description. Particles like photons are measured only occasionally, e.g., when they reach a detector. At the intermediate time, their quantum state becomes a superposition of localized states. Photons do interact with the environment between measurements, but only weakly, so that they are not localized in interferometers. Other particles, like neutrinos, interact only weakly most of the time.

In summary, a world consists of (a) macroscopic bodies and strongly interacting particles, which are frequently measured by the environment, (b) microscopic particles which are measured, or interact strongly, only sometimes, and (c) microscopic particles which are almost always coupled only weakly with the environment. Strongly interacting bodies (a) have a quantum state which is well localized at all times. The frequent measurements by the environment are nondemolition measurements and they create and measure both forwards and backwards evolving quantum states. Thus the backwards evolving state is identical to the time reversal of the forwards evolving state. Particles like photons (b) are measured occasionally, and mostly by demolition measurements. Strong measurements localize them, but in between measurements, their wave functions are typically spread out in space. Usually, their forwards and backwards evolving states are different: the backwards evolving state is not equal to the time reversal of the forwards evolving state, although it is never orthogonal to it. (Note that in some cases, several quantum particles are entangled and only their joint quantum state is pure.) Finally, weakly interacting particles (c) are spread out in space most of the time. The forwards and backwards evolving states need not be equal. Cosmology and experimental data tell us something about these states (mostly about the forwards evolving states).

Within a world, the objects of types (a), (b) and (c) influence one another and their states are causally connected. In the standard MWI, only forwards evolving states are considered. I will argue that the story of a world is much clearer if both forwards and backwards evolving states are considered at a particular time. The forwards evolving state of quantum particles on its own (or the set of measurement outcomes in the past which defines it) does not describe correctly the influence of the particles upon other objects. 


\section{Modified Everett worlds}

Here is the main point I'd like to make. The standard quantum state of a particle, which is defined at a particular moment of time, which evolves forwards in time, and which depends on macroscopic records of measurements performed in the past, is not enough to define the particle's influence on other systems in the world. We also need to know the backwards evolving state, i.e., the outcomes of future measurements.

I suggest, therefore, the following change to the MWI picture of the Universe. Instead of a tree of worlds, which starts from a single state common to all worlds and splits at every quantum measurement, the worlds are split by future measurements.

With this modification to the MWI, each Everett world is a possible Aharonov world. More precisely, it is a "clean" Aharonov world because at each moment in time, both the forwards and the backwards evolving states have only the relevant components $|\psi\rangle$ and $\langle\phi|$ corresponding to the particular results of complete measurements before and after the time in question. This contrasts with Aharonov's original proposal, where both forwards and backwards evolving states $|\Psi\rangle$ and $\langle\Phi|$ are superpositions. The particular components $|\psi\rangle$ and $\langle\phi|$ in these superpositions have only tiny absolute weights which, however, ensure dominant contributions when we calculate weak values or (counterfactual) probabilities using the ABL rule.

Let us look again at our example with a single particle source, beam splitter and two detectors (Figure 2). The world splits with the clicks of the detectors, creating a world in which the particle is reflected by the beam splitter and absorbed by detector $A$, and a world in which the particle passes through and is absorbed by detector $B$. In a standard approach to the MWI, at the time that the particle has passed the beam splitter, but has not yet reached a detector, there is only one world, with the particle in a superposition of two localized states. But nobody will ever observe any effect of this superposition (this is in contrast to an alternative experiment in which the beam splitter is part of a MZI). There will be two descendants of the experimenter who performs the experiment. One observes a click from detector $A$ and might see traces of weak coupling on the route $B S$ - $A$, and the other observes a click from detector $B$ and might see traces of weak coupling on the route $B S$ - $B$. The worlds, as I define them, are histories, one with the $A$ outcome and another with the $B$ outcome. In no world can a trace of the superposition of paths $A$ and $B$ can be seen, i.e., in no world 
will we see something both on the route $B S-A$ and on the route $B S-B$. In contrast, the traces of path $B S-A$ in world $A$ (and the traces of path $B S$ $B$ in world $B$ ) can be seen. To this end we have to arrange an ensemble of identical experiments with weak measurements of projection on various paths at the appropriate time. Then, the results of the weak measurements on a pre- and post-selected ensemble will show the trajectories $B S$ - $A$ and $B S-B$ respectively. (Especially effective is a weak measurement performed on a rare event pre- and post-selection ensemble when all particles in the ensemble end up with the desired outcome. Then, the same measuring device couples to all particles.)

The example with a single beam splitter is illustrative, but it is very simple, and although there is some inconsistency, we can also discuss it relatively well in the language of forwards evolving states only. Let us consider a more sophisticated example, in which the standard approach can lead to wrong conclusions [10]. Consider a combination of beam splitters which create a MZI "nested" in another MZI (see Figure 5). The internal MZI is tuned in such a way that a particle entering the left input port always comes out in the right port. We consider a photon which enters the external interferometer from the source and ends up in detector 2. Since photons on the right arm of the external MZI leave the interferometer (due to the particular tuning of the internal interferometer), in the framework of the standard MWI we would conclude that the photon passed solely through arm $A$ (and not through $B$ and $C$ ). However, such a setup of beam splitters can also be considered as the creation of the two-state vector (5) of the three box paradox, where $|A\rangle,|B\rangle$ and $|C\rangle$ are the states of a photon in arms $A, B$ and $C$ respectively [11]. Then, we know that the weak interaction influence at $B$ is as if the photon were there. In any case, it is not less than the influence at $A$. This fact is immediately seen in the world described by the two-state vector and is very hard to understand in the standard approach.

Aharonov's proposal is to add a Universal backwards evolving quantum state $\langle\Phi|$. My proposal is to add "locally" to each world, in the period between complete measurements on quantum particles, a backwards evolving quantum state of the particles $\langle\phi|$. Aharonov's backwards evolving state has a fundamental ontological status, similar to that of the forwards evolving universal state in the standard MWI. My additional backwards evolving state is not of this kind (at least until I introduce a more speculative modification below). It is an explanatory concept for the inhabitants of a particular world.

Sometimes in discussions of the meaning of the quantum state, it is con- 


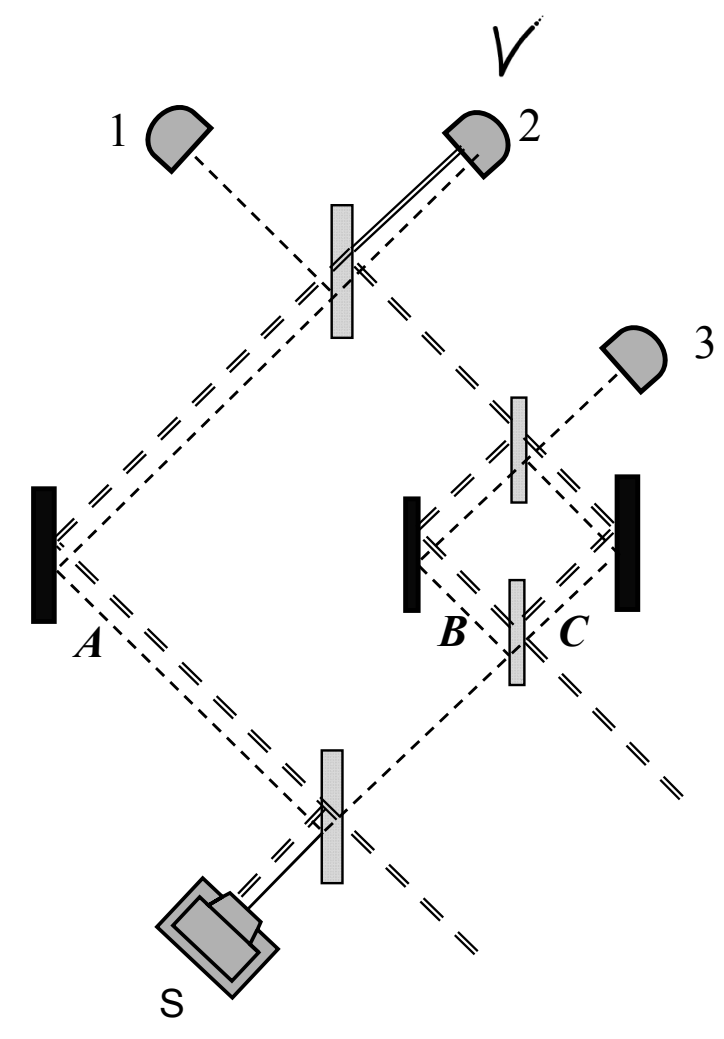

Figure 5: The internal Mach-Zehnder interferometer is tuned in such a way that the particle cannot go from source $S$ to detector 2 through the right arm. Nevertheless, at B both forwards and backwards evolving states are present, and for any weak coupling to the particle at B, the effect is as if there is a single particle there. 
sidered as a way to describe the information which belongs to a particular observer. In a particular world, at least for some periods in the past, forwards and backwards evolving states of particles describe correctly their influence on other objects. Note that the change is relevant only for objects of type (b); strongly interacting objects (a) have identical forwards and backwards evolving states and adding the backwards evolving state contributes nothing. Viewing the quantum state as information sometimes accompanies an attribution of ontological status to the outcomes of measurements. In this language, my proposal is to describe a quantum system at a particular time by the outcomes of measurement both before and after this time. Thus, the time of world splitting is the time of future measurements.

Let us clarify the concept of world splitting by returning to the experiment of Figure 2. A world is a complete history of results of measurements. When we talk about "splitting", we consider the following. We make, at consecutive times, snap shots of the Universe and count the number of worlds. The increase of this number signifies splitting. During the time in which the photon has left the source and is flying towards the detectors, the snap shots count two worlds: in one, the photon is detected (in the future) by detector $A$, and in the other by detector $B$. The number of worlds continues to be two also for snap shots performed after the time at which the photon reaches the detectors (the time of splitting according to the standard approach). Regarding the detectors we can see a change: when the photon reaches the detectors, their states become different in the two worlds. Before the time of arrival of the photon, both detectors are in the "ready state". It is the photon which distinguishes the worlds via its different ways of weakly coupling with the environment. Note that when we consider the Mach-Zehnder interferometer experiment (Figure 4), there are no different histories and there is a single world for snap shots at all times.

There is no paradox of backwards causality in this proposal, because the splitting of worlds is not a physical phenomenon: the concept of a "world" is given by our own semantic definition which helps to explain our experiences. The fundamental ontological picture remains, as in standard MWI, that of a single forwards evolving quantum state. The forwards evolving state of measuring devices defines the outcomes of measurements which, in turn, define the forwards and backwards evolving states within a world.

The time asymmetry in this picture is even worse than in Aharonov's proposal. In Aharonov's theory, the asymmetry is due to a very big difference in the form and role of the forwards and backwards evolving states $|\Psi\rangle$ and 
$\langle\Phi|$. In my picture, there is only one, forwards evolving, fundamentally ontological state. It defines, in a more or less symmetric way, the forwards and backward evolving states $|\psi\rangle$ and $\langle\phi|$ within various worlds, but on a global view there is an asymmetry: only about the forwards evolving states $|\psi\rangle$ can we say that their superposition is the Universal quantum state.

Can we restore time symmetry? Can we apply the TSVF globally and accept, as Aharonov does, the fundamental ontological existence of both forwards and backwards evolving quantum states? In the next section I will discuss such a possibility.

\section{Time symmetry}

The most natural way to combine the backwards evolving states of all branches leads to a backwards evolving universal state which is just the time reversal of the forwards evolving universal state. All other components of the backwards evolving states of different worlds interfere destructively. But it seems incorrect to assume that this is the fundamentally ontological backwards evolving quantum state of the Universe. The difficulty arises when we consider the issue of the probability of an outcome of a quantum experiment $[12]$.

In the MWI with a single ontological quantum state evolving forwards in time, we can postulate (or as David Deutsch claims [13], even derive) the observed Born rule for quantum mechanical probabilities. These are not "real" probabilities, they are a "measure of existence" or "caring measure" $[14,15]$, the main purpose of which is to advise a gambler how to bet on the outcomes of quantum measurements so as to gain a maximum reward for his multiple descendants. One can connect this measure to ignorance probabilities through a gedanken experiment involving a quantum gambler with a sleeping pill [16].

However, the assumption of identical ontological forwards and backwards evolving states leads to wrong probabilities: indeed, according to the ABL rule (2), the probability of an outcome $O=o_{n}$ is proportional to $\left|\left\langle\Psi\left|\mathbf{P}_{O=o_{n}}\right| \Psi\right\rangle\right|^{2}$, while according to the Born rule, the probability is proportional to $\left|\left\langle\Psi\left|\mathbf{P}_{O=o_{n}}\right| \Psi\right\rangle\right|$.

Apart from using the very special backwards evolving state proposed by Aharonov (in which the correct probabilities are fixed by fiat), we can obtain the correct probabilities if we assume that the backwards evolving state is a complete mixture, i.e., an equally weighted mixture of the states of an 
orthonormal basis.

This proposal might help us understand the main difficulty with the concept of probability in the MWI. In a quantum experiment all outcomes are realized, and so the standard concept - the probability that one outcome happens and not the others - evaporates. I had to introduce the "sleeping pill" trick to associate the probability of an outcome with the concept of probability understood as representing ignorance of the experimenter [16]. But now, when there is a fundamentally ontological mixture of states going backwards in time, corresponding to different outcomes of the experiment, we can associate the experimenter with both the forwards evolving state and a component of the backwards evolving mixture corresponding to one of the outcomes. For such an experimenter there is a matter of fact about the outcome of the experiment and the usual concept of probability applies.

Adding a backwards evolving quantum state which is a complete mixture is consistent with our observations, but it is only a small step towards time symmetry. The backwards and forwards evolving states are very different one is a mixture and the other is a pure state.

The modification which I have advocated here, with the backwards evolving state either ontological or not, has no observable effects. But it clarifies our concept of "world" and provides a step towards a better connection between the mathematical concepts of quantum mechanics and our experience.

It is a pleasure to thank Simon Saunders for suggesting the topic and helping tremendously in writing this paper. I also thank Jon Barrett for numerous helpful comments. This work has been supported in part by grant 990/06 of the Israel Science Foundation.

\section{References}

[1] Y. Aharonov and E. Gruss, Two-Time Interpretation of Quantum Mechanics, arXiv:quant-ph/0507269v1 (2005).

[2] Y. Aharonov and L. Vaidman, The Two-State Vector Formalism, an Updated Review, Lect. Notes Phys. 734, 399 (2008).

[3] L. Vaidman, Backward Evolving Quantum States, J. Phys. A 40, 3275 (2007). 
[4] Y. Aharonov and L. Vaidman, Properties of a Quantum System During the Time Interval Between Two Measurements, Phys. Rev. A 41, 11 (1990).

[5] Y. Aharonov, P. G. Bergmann and J. L. Lebowitz, Time Symmetry in the Quantum Process of Measurement, Phys. Rev. 134, B1410 (1964).

[6] Y. Aharonov, D. Z. Albert, and L. Vaidman, How the Result of Measurement of a Component of the Spin of a Spin- $\frac{1}{2}$ Particle Can Turn Out to Be 100, Phys. Rev. Lett. 60, 1351 (1988).

[7] L. Vaidman, Lorentz-Invariant "Elements of Reality" and the Joint Measurability of Commuting Observables, Phys. Rev. Lett. 70, 3369 (1993).

[8] L. Vaidman, Weak-Measurement Elements of Reality, Found. Phys. 26, 895 (1996).

[9] Y. Aharonov and L. Vaidman, Complete Description of a Quantum System at a Given Time, J. Phys. A 24, 2315 (1991).

[10] O. Hosten et al., Counterfactual Quantum Computation through Quantum Interrogation, Nature, 439, 949 (2006).

[11] L. Vaidman, Impossibility of the Counterfactual Computation for All Possible Outcomes, Phys. Rev. Lett. 98, 160403 (2007).

[12] S. Saunders, Time, Quantum Mechanics, and Probability, Synthese 114, 373 (1998).

[13] D. Deutsch, Quantum Theory of Probability and Decisions, Proc. Royal Soc. Lon. A 455, 3129 (1999).

[14] L. Vaidman, Many-Worlds Interpretation of Quantum Mechanics, Stan. Enc. Phil., E. N. Zalta (ed.) (2002), http://plato.stanford.edu/entries/qm-manyworlds/.

[15] H. Greaves, Understanding Deutsch's Probability in a Deterministic Multiverse, Stud. Hist. Phil. Mod. Phys. 35, 423 (2004).

[16] L. Vaidman, On Schizophrenic Experiences of the Neutron or Why We Should Believe in the Many-Worlds Interpretation of Quantum Theory, Int. Stud. Phil. Sci. 12, 245 (1998). 